\title{
An Analysis of Contrast Enhancement using Activation Functions
}

\author{
Gourav Garg ${ }^{1}$ and Poonam Sharma ${ }^{2}$ \\ Department of C.S.E. \& I.T. \\ Madhav Institute of Technology and Science, Gwalior, India \\ ${ }^{1}$ garg5656@gmail.com, ${ }^{2}$ enggpoonam@yahoo.com
}

\begin{abstract}
The contrast of an image is a feature which determines how image looks better visually. In this paper, we are analysing the capability of activation functions for contrast enhancement. Activation functions are classically used in neural network. In this paper, Activation function creates a mask which is operated on the image on pixel by pixel basis. On the basis of activation function the pixel value of image is changed which improves the contrast of image. We have used various activation functions such as sigmoid function, bipolar sigmoid function, RAMP function, hyperbolic tangent function. Contrast enhancement using these activation functions has been successfully apphed on several dark and bright images. For performance assessment we have used Peak Signal to Noise Ratio (PSNR), absolute mean brightness error (AMBE), and Structure Similarity Index (SSIM). From experimental result, it is obsened that RAMP function and hyperbolic tangent function have better image enhancement capability
\end{abstract}

Keywords: contrast enhancement, Histogram equiliation, sigmoidal function, bipolar sigmoidal function, hyperbolic tangent function, RAM function

\section{Introduction}

Image enhancement is a process which changes the pixel's intensity of the input image so as to make the outout mage looks better [1].Image enhancement technique has been proposed in many application of mage processing where subjective quality of image is most important and objective quality of image is depended on application circumstances. The aim of image enhaneement is to recover the interpretability or perception of information contained in the image for human viewers, or to deliver a "better" input for other automated image processing systems. Recently several algorithms have been published for contrast ephancement [1].

Contrast enhancement of an image is an important challenge in the field of digital image processing which is well-defined as the ratio between the brightest and the darkest pixel intensities of image.High contrast images contain much color and gray scale informa 101 as compare low contrast images.Contrast enhancement plays an important role in mage processing applications, such as medical image processing, digital photography, satellite imaging, and LCD display processing. There are several descriptions for an image to have poor contrast: due to the poor quality of the used imaging device. As a result, such images and videos may not expose all the details in the captured scene.

Contrast enhancement methods can be classified into two main classes: 1) intensitybased techniques and2) feature-based techniques [1].

Intensity-based methods can be explainedby the help of eq. (1).

$$
O(i, j)=f(I(i, j))
$$

WhereI( $i, j)$ is the original image, $O(i, j)$ is the output image after enhancement, and $f$ is a transformation function. 
The main idea of feature-based methods is to enhance the small-scale components, which represent some specified features, of an image and these methods can be explain as

$$
I_{0}(i, j)=L_{1}(i, j)+G(i, j) H_{1}(i, j)
$$

Where $\mathrm{H}_{1}$ represents the high frequency components which contain the concerned features, $\mathrm{L}_{1}$ represents the low frequency components which keep the basic appearance of the original image, $\mathrm{G}$ is the enhancement gain and $\mathrm{I}_{0}$ is output image.

Intensity-based methods are further classified into two main processes: 1) global histogram equalization (GHE) and 2) local histogram equalization (LHE). In GHE, the histogram of the whole input image is used to compute a histogram transformation function. As an outcome, the dynamic range of the image histogram is compressed and stretched, by which the overall contrast is enhanced [1]. The computational complexity of GHE is relatively low. The major drawback of GHE is that it cannot adjust the local information of the image and preserve the brightness of the original image. Whereas L HE uses a sliding window method, a window is set, to compute its local histogram. Then histogram equalization is applied to the local histogram to obtain the gray level transformation for the centric pixel. LHE is capable of generating good contrast results but is sometimes held to over-enhance images. It also requines more computation than other methods because a local histogram must be made and deal with for every image pixel.

Some extension methods of GHE have been developed for Contrast enhancement such as Limited Adaptive Histogram Equalization(CLAHE) [2], Brightness preserving bihistogram equalization (BBHE) [3], Dualistic sub-image histogram equalization (DSIHE) [4], Minimum mean brightness erron bi-h stogram equallization (MMBEBHE) [5], Recursive mean-separate histogram equalization (RMSHE) [6], Recursive sub-image histogram equalization (RSIHE) [7], Recursively Separated and Weighted Histogram Equalization for Brightness Preseryation and Contrast Enhancement(RSWHE)[8]. Nonparametric modified histogram equalisation for contrast enhancement (NMHE) [9], Brightness Preserving Dynamic Fuzzy Histogram Equalization [10].All these methods preserve brightness as wellas perform contrast enhancement.

In this paper, we use the activation function to create mask, this mask is applied on an input image to get a higher contrast image. We take four low contrast images and two dark images and applied mask on it. We found some results and compared it with the results of other methods by using peak signal noise to ratio (PSNR), absolute mean brightness error (AMBE) and structural similarity index (SSIM).

The structure of the paper is organized as follows: Section 2 presents Histogram equalization (HE) Th Section 3, describes various Activation functions. Section 4 discusses the activation function based algorithm. Section 5 represents performance evaluations. Section 6 results of various activation functions are reported. Section 7 concludes the paper.

\section{Histogram Equalization (HE)}

Many image enhancement methods have been proposed, one of most popular technique is histogram equalization (HE). This technique is usually used for image enhancement because of its simplicity, practical and comparatively better performance on almost all types of images [1]. Its basic idea depends on mapping the gray levels based on the probability distribution and cumulative density function of the input gray level.Applications of Histogram equalization is medical image processing and radar image processing. HE is rarely active in consumer electronic applications such as video surveillance, digital camera, and television since HE tends to familiarize some annoying artifacts and unnatural enhancement, including intensity saturation effect.

For a given image $\mathrm{X}$, the probability density function $\mathrm{p}\left(\mathrm{x}_{\mathrm{k}}\right)$ is defined as: 


$$
\mathrm{p}\left(\mathrm{X}_{\mathrm{k}}\right)=\frac{\mathrm{n}^{\mathrm{k}}}{\mathrm{n}}
$$

Where $n^{k}$ is no. of times that the level $x_{k}$ appears in input image $\mathrm{X}$ and $\mathrm{n}$ is total no. of pixel in the image.

Its cumulative distribution function (CDF) is defined as

$$
\mathrm{c}\left(\mathrm{x}_{\mathrm{k}}\right)=\sum_{\mathrm{i}=0}^{\mathrm{k}} \mathrm{p}\left(\mathrm{x}_{\mathrm{k}}\right)
$$

Where $\mathrm{k}=0,1 \ldots \mathrm{L}-1$, it is obvious that $\mathrm{c}\left(\mathrm{x}_{\mathrm{L}-1}\right)=1$ and $\mathrm{L}=255$ Based on the CDF, histogram equalization now maps an input gray level $x_{k}$ into an output gray level $f\left(x_{k}\right)$, where $\mathrm{f}\left(\mathrm{x}_{\mathrm{k}}\right)$, commonly called a level transformation function, is defined as

$$
f\left(x_{k}\right)=x_{0}\left(x_{L-1}-x_{0}\right) \cdot c\left(x_{k}\right)
$$

HE can introduce a significant change in brightness of an Image, which can be applied to the direct application of HE scheme in consumer electronics.

\section{Activation Function}

A function used to transform the activation level of a unit (neuron) into an output signal. Typically, activation functions have a "squashing" effect. Neural Networks supports a wide range of activation functions. Onky a few of these are used by default; the others are available for customization. Some of the most widely used activation functions solve non-linear problems. These functions are: Unipolar sigmoid, Bi-polar sigmoid, hyperbolic tangent and Ramp function. We did not care about some activation functions such as identity function, step function or binary step function as they are not used to solve linear problens.

\subsection{Uni Sigmoid Function}

Sigmoid function is a honstop nonlinear activation function. The name, sigmoid, acquired from the fact that the fumction is "S" shaped $[11,12,14]$. Mathematicians call this function the logistic function, $\mathrm{f}(\mathrm{x})$ is the sigmoid function which is defined as:

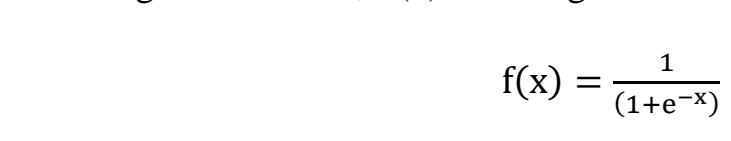

The sigmoid function has the looks that it is a smooth continuous function, the function outputs within the ange 0 to 1 , Figure 1 is illustrates the sigmoid function[12].
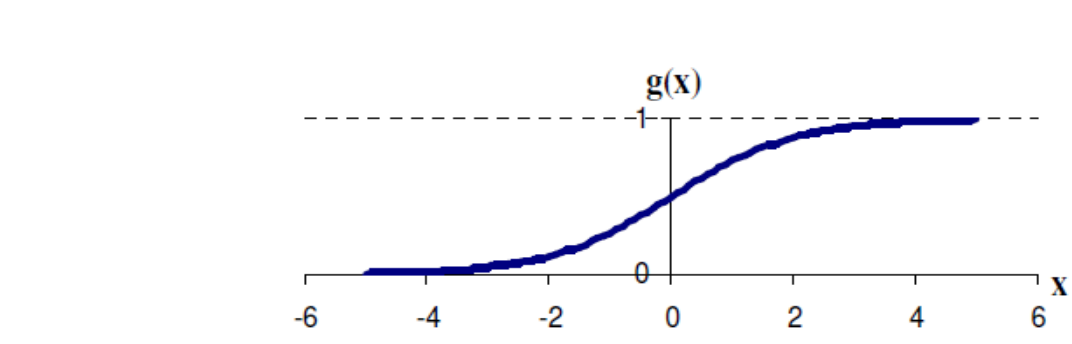

Figure 1. Unipolar Sigmoid Function

\subsection{Bi-polar Sigmoid}

This function is another type of sigmoid function, Bi-polar sigmoid function is an extension method of unipolar sigmoidal function, and it is given by $[11,12]$ 


$$
f(x)=\frac{\left(1-e^{-x}\right)}{\left(1+e^{-x}\right)}
$$

This function is similar to the sigmoid function, but range of output function is $[-1$, 1].Bipolar sigmoid function is described in Figure 2 [12].

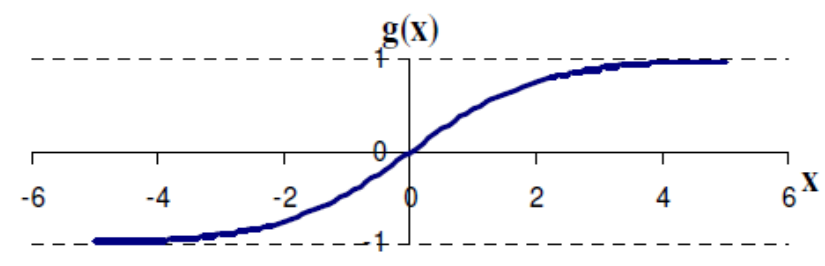

Figure 2. Bi-polar Sigmoid Function

\subsection{Hyperbolic Tangent Function}

This function is defined as the ratio between the hyperbolic sine and cosine functions or expanded as the ratio of the half-difference and half-sum of two exponential functions in the points $\mathrm{x}$ and $-\mathrm{x}$ as follows:

$$
\tanh (x)=\frac{\sinh (x)}{\cosh (x)}=\frac{e^{x}-e^{-x}}{e^{x+e^{-x}}}
$$

This function is similar to the sigmoid function, burange of output function is $[-1,1]$. Hyperbolic tangent function is described in Figure 3 112.

\subsection{Ramp Function}

Ramp Function $\mathrm{f}(\mathrm{x})$ states that the signal will start from time zero and instantly will take a slant shape and depending upon characteristics and input(either positive or negative) the signal will follow the straight slant path either towards right or left, here towards right. Ramp function is given by [13].

$$
f(x)= \begin{cases}x & \text { if } x \geq 0 \\ 0 & \text { if } x<0\end{cases}
$$

The range of output function is [0, 1]. Ramp function is described in Figure 4 [12].

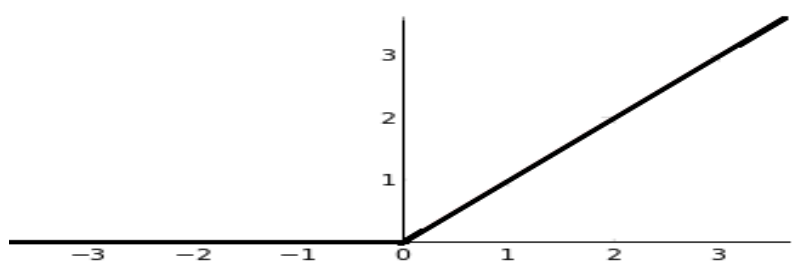

Figure 4. Ramp Function 


\section{Activation function based algorithm}

It is a point process method that is executed directly on each pixel of an image, independent of all other pixels in the image to change the dynamic range. The mask which is applied on the input images is a non-linear activation function, which is obtained by multiplying the activation function with the input and by a factor $\mathrm{C}$.

Suppose mask of sigmoidal function is defined in equation (10). We used that factor to determine the most wanted degree of the contrast depending on the degree of darkness or brightness of the original image.

$$
\text { Mask }=\mathrm{I}(\mathrm{i}, \mathrm{j}) * \mathrm{C} * \mathrm{ac}
$$

Where ac is determine the activation function in equation (10).The mask is passed over the image from image's upper left corner pixel by pixel. Each pixel's intensity value of output image is equal to pixel's intensity value of input image added to the value of the mask.

The intensity value of output image is given by

$$
\mathrm{O}(\mathrm{i}, \mathrm{j})=\mathrm{I}(\mathrm{i}, \mathrm{j})+\text { Mask }
$$

Where $\mathrm{O}(\mathrm{i}, \mathrm{j})$ is processed image, $I(\mathrm{i}, \mathrm{j})$ is unprocessed input image and $\mathrm{C}$ is a contrast factor which determines the degree of the needed contrast.

The value of $\mathrm{C}$ depends on the objective of the enhancement process, generally value of $\mathrm{C}$ is one, two or three, the user can select the value of $\mathrm{C}$ according to desired contrast that he needs. The pixel values are within a limited range $(0-255)$ for an 8-bit image. In this paper we generally use of value of $\mathrm{C}$ astwofor low contrast images and as one for dark images.

\section{Performance Evaluation}

Performance evaluation is done on severalow contrast images. Four low contrast images and two dark images have been aken as input for evaluating the results. Visual results have been displayed and quantitative comparison has been done on the basis of (a) peak signal-to-noise ratio (b) Absolute mean brightness error(AMBE) (c) structure similarity index.

Mathematical Formulas for Quality Factors is as follows:

\subsection{Peak Signd to Noise Ratio (PSNR)}

Assume that $N$ is the total number of pixels in the input or output image, MSE (Mean Squared Error) is calculated through (12). Based on MSE, we calculate PSNR then it is defined as (13). According to the definition of PSNR the output image quality is better if those image has maximum PSNR.

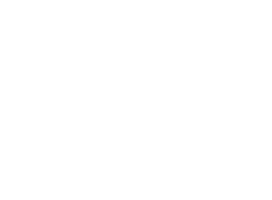

$$
\begin{gathered}
\mathrm{MSE}=\frac{\sum_{\mathrm{I}} \sum_{\mathrm{J}}\left(\mathrm{x}_{\mathrm{i} . \mathrm{j}}-\mathrm{y}_{\mathrm{i}, \mathrm{j}}\right)^{2}}{\mathrm{~N}} \\
\text { PSNR }=10 \log _{10} \frac{255^{2}}{\mathrm{MSE}}
\end{gathered}
$$

\subsection{Absolute Mean Brightness Error(AMBE)}

It determines the value of error in between processed image and input image. Suppose $X_{M}$ is the mean of the input image $\mathrm{X}=\left\{x_{i . j}\right\}$ and $Y_{M}$ is the mean of the output image $\mathrm{Y}=$ $\left\{y_{i, j}\right\}$. Minimum AMBE value is desired which is obtained when output image have almost same mean as the mean of the input image.

$$
\operatorname{AMBE}(\mathrm{X}, \mathrm{Y})=\left|\mathrm{X}_{\mathrm{M}}-\mathrm{Y}_{\mathrm{M}}\right|
$$




\subsection{Structure Similarity Index (SSIM)}

SSIM is a method for calculating the similarity between two images. Value of SSIM varies in between -1 and 1 .

$$
\operatorname{SSIM}=\frac{\left(2 \mu_{\mathrm{x}} \mu_{\mathrm{y}}+\mathrm{c}_{1}\right)\left(2 \sigma_{\mathrm{xy}}+\mathrm{c}_{2}\right)}{\left(\mu_{\mathrm{x}}^{2}+\mu_{\mathrm{y}}^{2}+\mathrm{c}_{1}\right)\left(\sigma_{\mathrm{x}}^{2}+\sigma_{\mathrm{y}}^{2}+\mathrm{c}_{2}\right)}
$$

Where $\mu_{x}$ is the average of input image $\mathrm{X}=\left\{\mathrm{X}_{\mathrm{i} . \mathrm{j}}\right\}$ and $\mu_{y}$ is the average of output image $\mathrm{Y}=\left\{\mathrm{y}_{\mathrm{i}, \mathrm{j}}\right\} . \sigma_{\mathrm{x}}^{2}, \sigma_{\mathrm{y}}^{2}$ is variance of input and output image respectively. $\sigma_{\mathrm{xy}}$ is covariance between input and output image, Value of $c_{1}$ and $c_{2}$ is defined as. $c_{1}=$ $\left(\mathrm{k}_{1} \mathrm{~L}\right)^{2}, \mathrm{c}_{1}=\left(\mathrm{k}_{2} \mathrm{~L}\right)^{2}$ where $\mathrm{k}_{1}=0.01, \mathrm{k}_{2}=0.03$ (by default). And $\mathrm{L}$ is a dynamic range of pixel value.

\section{Results and Discussion}

We have considered four images of low contrast and last two images are dar $\mathrm{k}$, for better performance we take value of $\mathrm{C}$ as 2 for low contrast images and 1 fo high contrast images. All images are evaluated in terms of PSNR, AMBE, and SSIM.

Table 1. PSNR of Various Images

\begin{tabular}{|c|c|c|c|c|}
\hline Images & Ui-sigmoidal & $\begin{array}{c}\text { Bipolar } \\
\text { sigmoidal }\end{array}$ & RAMP & $\begin{array}{c}\text { Hyperbolic } \\
\text { Tangent }\end{array}$ \\
\hline Lena & 18.5152 & 11.9896 & 23.5292 & $\mathbf{2 3 . 8 4 4 9}$ \\
\hline Lady with hat & 13.4670 & 12.5676 & 13.0647 & $\mathbf{1 6 . 1 4 6 2}$ \\
\hline Tree & 11.9704 & 13.1831 & 14.0095 & $\mathbf{1 4 . 3 0 0 2}$ \\
\hline Building & 18.7018 & 13.0341 & 17.8824 & $\mathbf{1 9 . 8 0 8 8}$ \\
\hline Man with balloon & 15.4910 & 22.4102 & $\mathbf{2 5 . 1 5 3 6}$ & 16.0482 \\
\hline Flower & 14.9890 & 17.0131 & $\mathbf{1 7 . 8 0 6 9}$ & 14.2345 \\
\hline Average & 15.5224 & 15.0329 & $\mathbf{1 8 . 5 7 4 4}$ & 17.3971 \\
\hline
\end{tabular}

Table 2. AMBE of Various Images

\begin{tabular}{|c|c|c|c|c|}
\hline Images & Ui-sigmidail & $\begin{array}{c}\text { Bipolar } \\
\text { sigmoidal }\end{array}$ & RAMP & $\begin{array}{c}\text { Hyperbolic } \\
\text { Tangent }\end{array}$ \\
\hline Lena & 29.3718 & 64.1087 & $\mathbf{2 . 9 2 9 1}$ & 11.9610 \\
\hline Lady with hat & 50.1593 & 59.4222 & 55.4594 & $\mathbf{2 1 . 3 2 8 8}$ \\
\hline Tree & 61.5178 & 55.4211 & 49.8155 & $\mathbf{4 0 . 3 0 1 5}$ \\
\hline Building & 24.0540 & 56.1167 & $\mathbf{5 . 6 6 2 0}$ & 14.1721 \\
\hline Man with bălloon & 27.9040 & 11.7973 & $\mathbf{4 . 9 3 9 3}$ & 26.6602 \\
\hline Flower & 35.4318 & 27.5365 & $\mathbf{2 1 . 7 9 4 9}$ & 40.3647 \\
\hline Ayerage & 38.0731 & 45.7337 & $\mathbf{2 3 . 4 3 3 5}$ & 25.7980 \\
\hline
\end{tabular}

Table 1 represents performance of various enhancement techniques on test image Lena, Einstein, peppers and house in terms of PSNR. We observe that RAMP and hyperbolic function produce higher PSNR values than other existing methods in the Table 1. Figure 5 shows the graphical representation of Table 1. Comparison of activation function is term of PSNR of various images.

Table 2 represent various techniques on the same image in term of AMBE, value of AMBE in RAMP function and hyperbolic function is less than other existing methods. The basis of above results RAMP and hyperbolic tangent function are best in comparison to sigmoid and bipolar sigmoid function. For the AMBE quantitative test, obviously the sigmoid, and unipolar sigmoid methods fail to preserve the original mean brightness. 
Further these two methods tend to produce intensity saturation and information loss. Figure 6 shows the graphical represent of Table 2.

Table 3. SSIM of Various Images

\begin{tabular}{|c|c|c|c|c|}
\hline Images & Ui-sigmoidal & Bipolar sigmoidal & RAMP & $\begin{array}{c}\text { Hyperbolic } \\
\text { Tangent }\end{array}$ \\
\hline Lena & $\mathbf{0 . 9 5 6 5}$ & 0.7686 & 0.9243 & 0.9492 \\
\hline Lady with hat & $\mathbf{0 . 9 1 8 6}$ & 0.8552 & 0.8593 & 0.8846 \\
\hline Tree & 0.9072 & 0.9028 & $\mathbf{0 . 9 0 7 9}$ & 0.8698 \\
\hline Building & $\mathbf{0 . 9 5 0 8}$ & 0.6503 & 0.8372 & 0.8661 \\
\hline $\begin{array}{c}\text { Man with } \\
\text { balloon }\end{array}$ & 0.9361 & 0.9366 & $\mathbf{0 . 9 3 7 1}$ & 0.9086 \\
\hline Flower & $\mathbf{0 . 8 8 3 6}$ & 0.7599 & 0.7572 & 0.7418 \\
\hline Average & $\mathbf{0 . 9 2 5 6}$ & 0.8122 & 0.8705 & 0.8701 \\
\hline
\end{tabular}

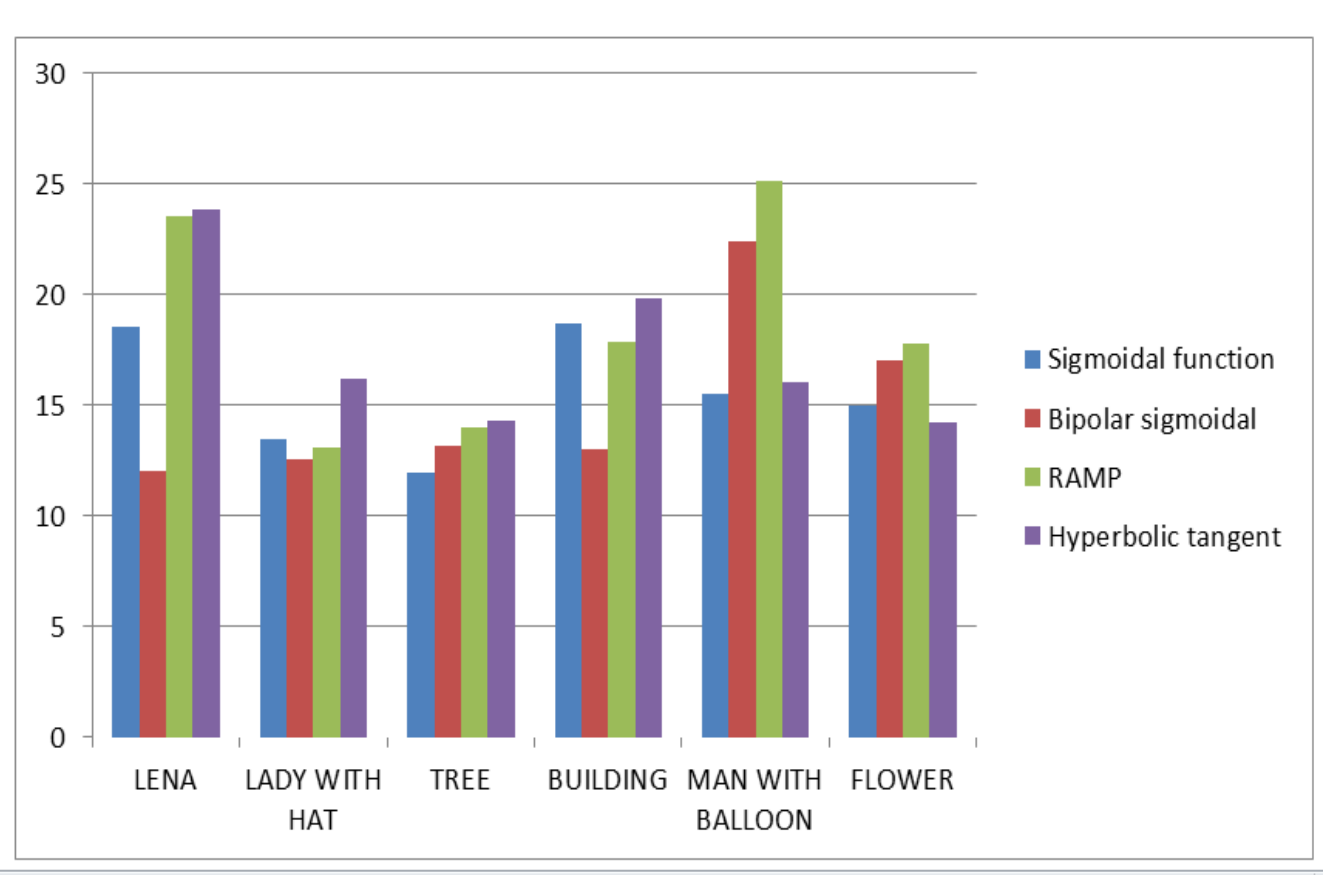

Figure 5. Bar Graph of PSNR

Table 3 presents the value of SSIM of various methods on same image. The SSIM represent similarity of two images. The values of sigmoid function in Table 3 are approximately are same. It is evident from the visual comparison that sigmoid function is similar to original image, but contrast of sigmoid function does not increases. "An Analysis of Contrast Enhancement using Activation Functions" observes that RAMP and hype bolic tangent are better method for enhancing the contrast. Figure 7 shows the graphical representation of Table 3.

Figure 8 shows the outcome of using various activation functions on image of Lena, Lady with hat, Tree, building, Man with balloon and Flower. In visual analysis it is observed that contrast has been enhanced to various levels by all the method but RAMP and hyperbolic tangent function are enhancing the image more precisely in comparison to sigmoid and bipolar sigmoid function. 


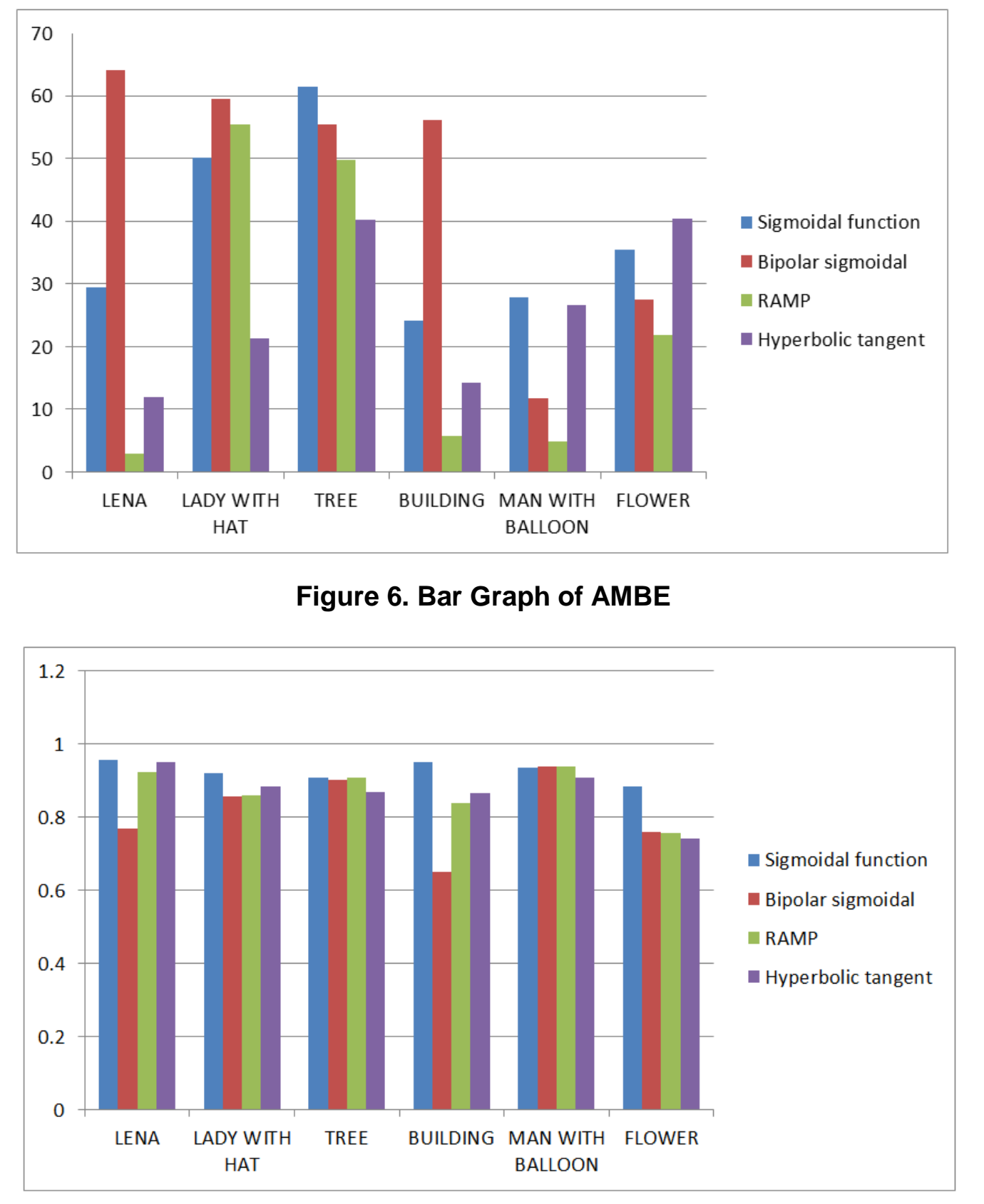

Figure 7. Bar Graph of SSIM

\section{Conclusion}

In this paper, we presented a new \& quite simple brightness contrast method that uses the activation function for calculating the mask; this mask is applied on original image in order to improve the overall dynamic appearance of an image. We have used four conventional differentiable and monotonic activation functions for comparison. After the result analysis, we found that RAMP and hyperbolic tangent are better in performance compared to sigmoid and bipolar sigmoid. 


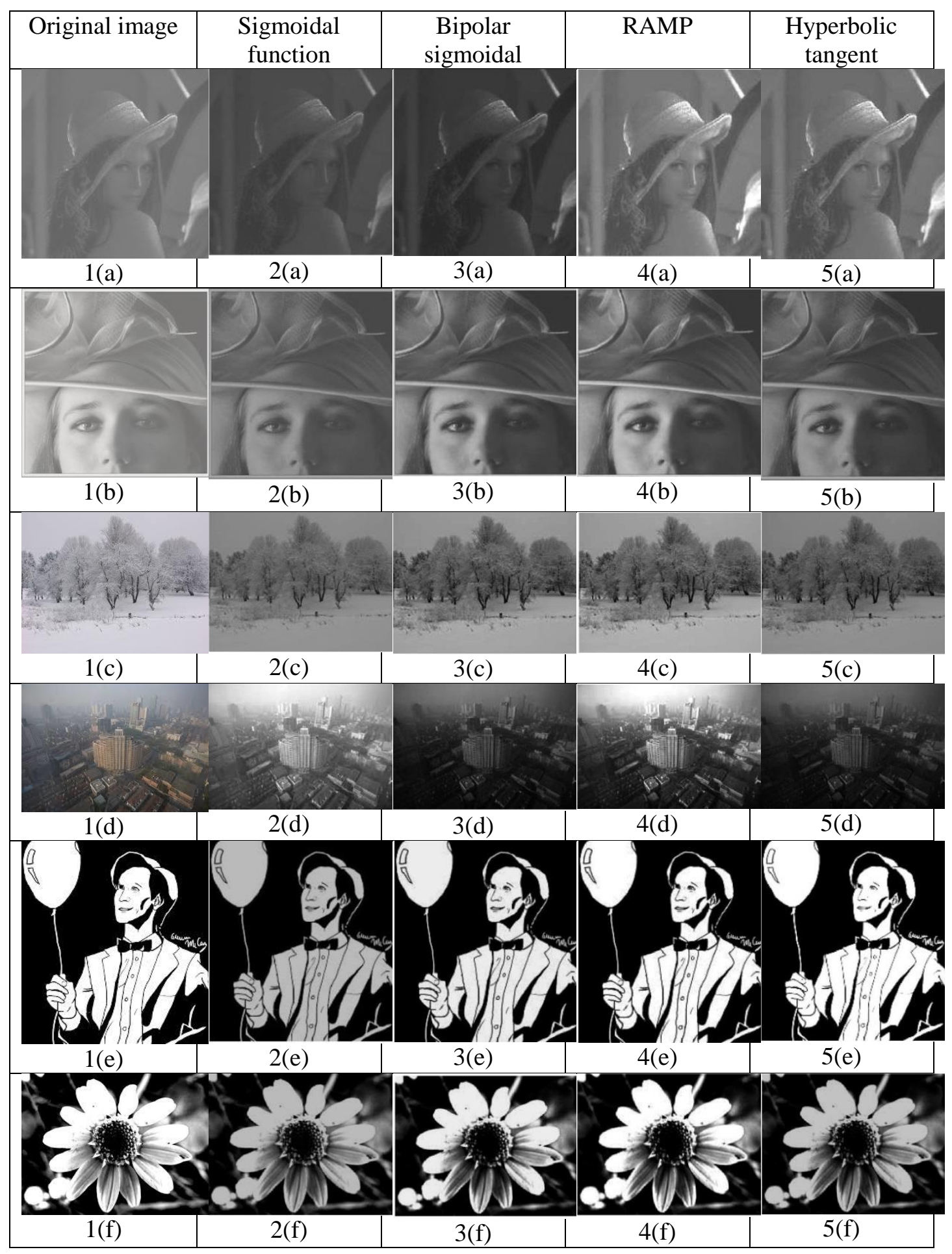

Figure 8. 1(a-f) Represent Original Test Images of Lena, Lady with hat, Tree, Building, Man with Balloon and Flower Respectively, 2(a-f) Represent Enhance by Sigmoid, 3(a-f) Represent Enhance by Bipolar Sigmoid, 4(a-f) Represent Enhance by RAMP , 5(a-f) Represent Enhance by Hyperbolic Tangent

\section{References}

[1] Rafael C. Gonzalez, and Richard E. Woods, "Digital Image Processing," Third Edition Prentice Hall. 
[2] S.M. Pizer, and R. E. Johnston, "Contrast limited adaptive histogram equalization: speed and effectiveness," IEEE Transl. on Consumer Electronics, 1990.

[3] Y. T. Kim, "Contrast enhancement using brightness preserving bi histogram equalization," IEEE Transl. on Consumer Electronics, vol. 43, no.1, February 1997.

[4] Y. Wang, Q. Chen, and B. Zhang, "Image enhancement based on equal area dualistic sub image histogram equalization method," IEEE Transl. on Consumer Electronics, vol.45, no.1 pp. 68-75, February 1999.

[5] S. Chen, and A. R. Ramli, "Minimum mean brightness error bi-histogram equalization in contrast enhancement," IEEE Transl. on Consumer Electronics, vol. 49,no. 4, pp. 1310-1319, November 2003.

[6] S. Chen, and A. R. Ramli, "Contrast enhancement using recursive mean-separate histogram equalization for scalable brightness preservation," IEEE Transl. on Consumer Electronics, vol. 49,no. 4, pp. 13011309, November 2003.

[7] K. S. Sim, C.P. Tso, and Y.Y. Tan, "Recursive sub-image histogram equalization applied to gray-scale images," IEEE Transl. on Consumer Electronics, vol. 28, pp. 1209-1221, July 2007.

[8] M. Kim, and M. G. Chung, "Recursively separated and weighted histogram equalization for brightness preservation and contrast enhancement," IEEE Transl. on Consumer Electronics, vol. 54, no. 3, pp. 13891397, August 2008.

[9] S. Poddar, and S.Tewary, "Non-parametric modified histogram equalisation for contrastenhancement," IEEE Transl. on Consumer Electronics, vol. 7, no. 7, pp. 641-652, August 2013.

[10] D.Sheet, and H.Garud, "Brightness preserving dynamic fuzzy histogram equalization," IEEE Transl. on Consumer Electronics, vol. 56, no. 5, November 2010.

[11] N. Hassan, and N. Akamatsu, "A new approach for contrast enhancement using sigmoid function," International Arab Journal of Information Technology, vo1.1, no. 2, July 2004.

[12] B. Karlik, and A. V. Olgac, "Performance analysis of various activation functions ingeneralized MLP architectures of neural networks," International Journal of Artificial Intelligence and Expert Systems (IJAE), vol.1 no. 4.

[13] S. Singh, "Proposed Concept of Signals fôr Ram Functions," World Congress on Engineering and Coputer Science, vol. 1, pp. 20-22, october 2010.

[14] Saruchi, "Adaptive sigmoid function to enhancement tow contrast imaget," International Journal of Computer Application, vol. 55, no. 4 p October 2012.
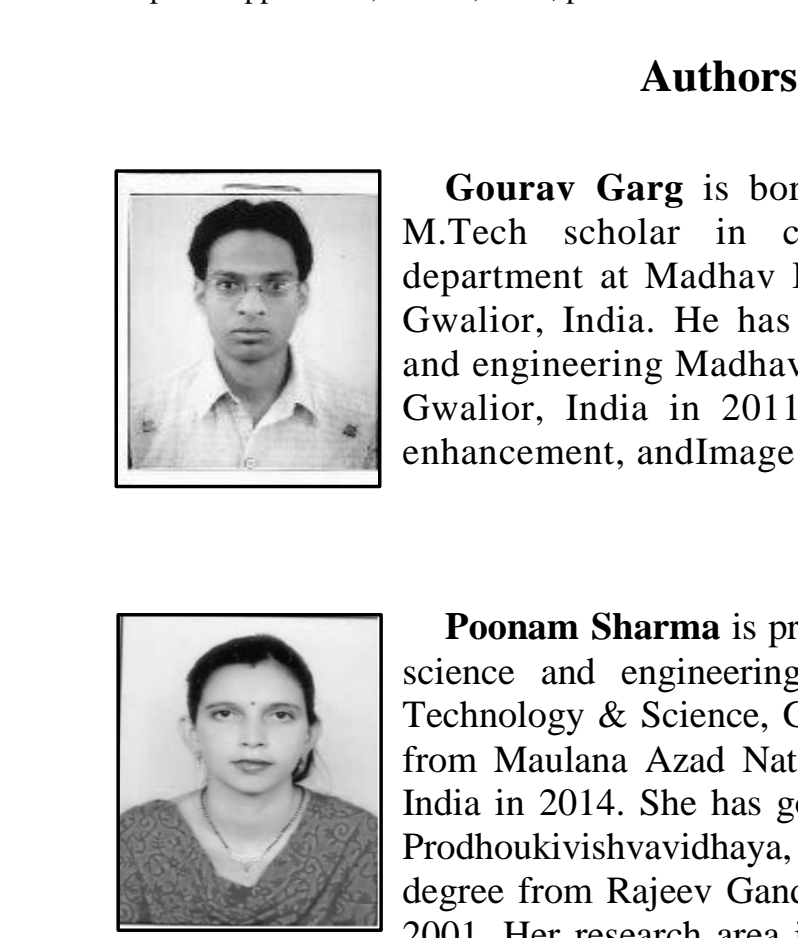

Gourav Garg is born in India in 1987. He is currently M.Tech scholar in computer science and engineering department at Madhav Institute of Technology and Science, Gratior, India. He has completed B.E in computer science and-ngineering Madhav Institute of Technology and Science, Gwalior, India in 2011. His research area includes Image enhancement, andImage denoising.

Poonam Sharma is presently Assistant Professor in computer science and engineering department at Madhav Institute of Technology \& Science, Gwalior, India. She has got PhD degree from Maulana Azad National Institute of Technology, Bhopal, India in 2014. She has got M.Tech degree from Rajeev Gandhi Prodhoukivishvavidhaya, Bhopal in 2005. She has got B.E. degree from Rajeev Gandhi Prodhoukivishvavidhaya, Bhopal in 2001. Her research area includes Biometrics, Image processing, soft computing and pattern recognition. 